\title{
Automedicación en personas adultas que acuden a boticas del distrito Jesús Nazareno, Ayacucho 2015
}

Self-medication in adults attending the drugstores of the Jesús Nazareno District,

Ayacucho 2015

\author{
Mery Luz Pillaca-Medina ${ }^{1,2}$, Kristian Carrión-Domínguez ${ }^{3,4}$ \\ ${ }^{1}$ Magister en Gerencia en Servicios de Salud, Universidad Nacional San Cristóbal de Huamanga. \\ ${ }^{2}$ Dirección General de Medicamentos, Insumos y Drogas, Ministerio de Salud, Lima, Perú. \\ ${ }^{3}$ Químico Farmacéutico de la Universidad Nacional San Cristóbal de Huamanga, Ayacucho, Perú. \\ ${ }^{4}$ Dirección Regional de Medicamentos, Insumos y Drogas, Dirección Regional de Salud, Ayacucho, Perú.
}

\begin{abstract}
Resumen
Introducción. La automedicación es un problema serio que afecta la salud de la población. Los estudios demostraron que hay relación entre la automedicación y los factores sociodemográficos y culturales. Objetivo. Determinar la frecuencia y las características de la automedicación en personas adultas que acuden a las boticas. Diseño. Estudio descriptivo y transversal. Lugar. Distrito Jesús Nazareno (DJN), Ayacucho, Perú. Intervenciones. Previo consentimiento informado, se realizaron encuestas a personas adultas que acudieron a 22 boticas durante setiembre a diciembre de 2015. La muestra fue probabilística aleatoria simple, conformada por 433 personas cuyas edades fluctuaron entre 25 a 65 años. La encuesta fue aprobada por el Comité Institucional de Ética en Investigación del Instituto Nacional de Salud. Los datos fueron procesados en el programa estadístico SPSS 19.0. Resultados. La frecuencia de automedicación fue $(87,8 \%)$. Las mujeres $(67,9 \%)$ se automedicaron más que los varones, ambos con estudio superior $(42,9 \%)$. E principal problema de salud fue el dolor $(64,7 \%)$. Los encuestados consideraron que su enfermedad era leve $(92,1 \%)$ y esperaron demasiado tiempo en los servicios de salud $(18,7 \%)$. Las personas que fueron recomendadas por un técnico representaron el 69,4\%, mientras que las personas influidas por la televisión, 74,7\%; principalmente usaron los analgésicos (64,7\%). Conclusiones. La automedicación en personas adultas del DJN fue alta, se presentó en ocho de cada diez personas y con estudios de nivel superior; predominó el sexo femenino. El principal problema de salud fue el dolor. El medicamento más usado fue el analgésico, el cual fue requerido principalmente porque las personas consideraron que su enfermedad era leve. Asimismo, un porcentaje considerable señaló que en los servicios de salud el tiempo de espera es prolongada.
\end{abstract}

Palabras clave. Automedicación; Adultos; Medicamentos; Perú.

\section{Abstract}

Introduction. Self-medication is a serious problem that affects population health. Studies show that there is relation between selfmedication and sociodemographic and cultural factors. Objective. To determine the frequency and characteristics of self-medication in adults who go to the pharmacies. Design. Descriptive cross-sectional study. Setting. Jesus Nazareno District (JND), Ayacucho, Peru. Interventions. Prior informed consent surveys were conducted on adults who attended 22 drugstores from September to December 2015. The sample was a simple probabilistic random one, formed by 433 people 25 to 65 years old. The survey was approved by the Institutional Research Ethics Committee of the Peruvian National Institute of Health. Data were processed with the statistical program SPSS 19.0. Results. The frequency of self-medication was $87.8 \%$. Women (67.9\%) self-medicated more than men, both had higher education (42.9\%). The main health problem was pain (64.7\%) and respondents felt that their disease was mild $(92.1 \%)$ and they had to wait too long in health services (18.7\%). $69.4 \%$ of people had received advice from a health technician, and $74.7 \%$ had been influenced by television. Respondents mainly consumed analgesics (64.7\%). Conclusions. The frequency of self-medication in adults from JND was high; it presented in eight out of ten people with higher education, and females predominated. The main health problem was pain. The type of drug most commonly used were painkillers, mainly because people felt that their illness was mild, and considered the waiting time in health services was too long.

Keywords. Self-medication; Adults; Medicines; Peru.

An Fac med. 2016;77(4):387-92 / http://dx.doi.org/10.15381/anales.v77i4.12652

\section{INTRODUCCIÓN}

La automedicación es un tema de interés universal y muy común en nuestra sociedad. Aunque la administración adecuada de medicamentos tiene el potencial de conferir beneficios, también pueden ocasionar problemas en la salud de la población. Esta práctica podría generar una identificación inadecuada del problema de salud, debido a elección, uso y administración incorrectos del medicamento, así como dependencia, reacciones adversas y asociaciones peligrosas en pacientes en riesgo.

La automedicación, según la OMS "Es la selección y uso de los medicamentos por parte de las personas sin prescripción médica, con el propósito de tratar enfermedades o síntomas que ellos mismos pueden identificar", mientras que la automedicación responsable la define como "Una práctica mediante la cual las personas tratan sus dolencias y afecciones con el uso de medicamentos autorizados, disponibles sin necesidad de prescripción, y que son seguros 
y eficaces si se los emplean según las indicaciones" (1). La Autoridad Nacional de Medicamentos (ANM) realiza campañas restringidas contra la automedicación, por lo cual la gran mayoría de la población no accede a una información que le permita tomar una decisión responsable para automedicarse.

En la Ley General de Salud, artículo $26^{\circ}$, se señala: "Solo los médicos, pueden prescribir medicamentos, los cirujanos dentistas y las obstetrices podrán prescribir dentro del área de su profesión". El artículo $33^{\circ}$ establece que: "El profesional químico-farmacéutico es el responsable de la dispensación, información y orientación al usuario sobre la administración, uso y dosis del producto farmacéutico..." (2). La ANM clasifica los productos farmacéuticos, dispositivos médicos y productos sanitarios para efectos de su dispensación en cuatro categorías: venta con receta especial numerada, venta con receta médica en establecimientos farmacéuticos (EF), venta sin receta médica exclusiva en EF y en establecimientos comerciales ${ }^{(3)}$.

Distintos estudios a nivel mundial muestran esta problemática. Según la Oficina de Política Nacional de Control de Drogas de Estados Unidos, se han producido más de 37 mil muertes por sobredosis de medicamentos al año. En Reino Unido, el analgésico provocó alrededor de 121 muertes anuales por intoxicación. La Asociación Argentina de Propaganda Médica estimó que 126 mil personas fueron hospitalizadas por año a causa de la automedicación ${ }^{(4)}$.

El desempleo, falta de acceso, escasa disponibilidad de información, deficiente educación sanitaria, así como la intervención de los familiares y amigos que ofrecen una alternativa basada en su propia experiencia constituyen factores prevalentes de la automedicación, como lo demuestra varios estudios realizados en el Perú. En Lima, el 58\% de los usuarios del Hospital Nacional Arzobispo Loayza se automedicó ${ }^{(5)}$. En Amazonas, la automedicación representó el 60,03\% de personas adultas ${ }^{(6)}$.
Las investigaciones realizadas por Consumers International demostraron que la industria gasta el doble en promoción y publicidad que en investigación y desarrollo, siendo la automedicación incentivada por laboratorios a través de estímulos otorgados a las farmacias ${ }^{(7)}$.

El objetivo del estudio fue determinar la frecuencia y las características de la automedicación en personas adultas que acudieron a las boticas del distrito Jesús Nazareno (DJN).

\section{MÉTODOS}

Investigación de tipo descriptivo y transversal. La población de estudio estuvo constituida por 5910 personas adultas con edades entre 25 y 65 años de ambos sexos del DJN. El criterio de inclusión fueron personas que acudieron a boticas y que vivían en el DJN. Según la base de datos de la Dirección Regional de Medicamentos, Insumos y Drogas - Diremid Ayacucho (2015), en el DJN se registraron 22 boticas, este total fue considerado para el presente trabajo.

Se calculó la muestra con un nivel de confianza del $95 \%$ y se consideró la proporción de individuos en la población con características estudiadas $(p=0,5)$ y para la proporción de individuos que no poseían esa característica $(q=1-p)$. El error estimado fue de $5 \%$ con una tasa de no respuesta de $20 \%$.

La muestra obtenida fue probabilística aleatoria simple, de acuerdo a la fórmula de tamaño muestral, y estuvo conformada por 433 personas adultas que acudieron a 22 boticas del DJN, durante el periodo de setiembre a diciembre de 2015.

Para la recolección de datos, se utilizó un cuestionario estructurado, compuesto por 17 ítems, con preguntas dicotómicas de opción múltiple, distribuidas en cuatro secciones: antecedentes de las personas, razones para la automedicación, tipo de medicamentos usados y problemas de salud. La en- cuesta fue aprobada por el Comité Institucional de Ética en Investigación del Instituto Nacional de Salud. Los participantes del estudio firmaron el consentimiento informado después de conocer los aspectos esenciales de la investigación.

Los datos fueron procesados usando el programa estadístico SPSS 19.0, a fin de realizar la estadística descriptiva, promedios, porcentajes, análisis e intervalos de confianza al 95\%.

\section{RESULTADOS}

La frecuencia de automedicación correspondió a $87,8 \%$. Del total de 433 personas, el $67,9 \%$ fueron mujeres y $32,1 \%$, varones. En general, esta práctica fue mayor en personas adultas con edades entre 25 a 34 años $(42,4 \%)$ y en personas con estudios superiores (42,9\%). Hubo diferencia significativa entre la automedicación y el grado de instrucción.

La mitad de ellos tenía trabajo independiente. El $61,1 \%$ tenía un ingreso mensual menor que el mínimo vital.

El $92,1 \%$ de adultos percibió que su enfermedad era leve. El 77,4\% de 380 encuestados alegó que se automedicó por recomendaciones de otras personas, siendo los técnicos en salud las personas que más influyeron en la población de estudio $(69,4 \%)$. La televisión fue el medio más influyente en la automedicación (74,7\%).

Los medicamentos más usados fueron los analgésicos $(64,7 \%)$ y antihistamínicos (56,3\%). El problema de salud más común fue el dolor $(64,7 \%)$. Los diez medicamentos más comúnmente utilizados fueron: acetaminofén 500 $\mathrm{mg}$, ibuprofeno $400 \mathrm{mg}$, naproxeno 550 $\mathrm{mg}$, acetaminofén $500 \mathrm{mg}+$ maleato de clorfeniramina $2 \mathrm{mg}+$ clorhidrato de fenilefrina $5 \mathrm{mg}$, maleato de clorfenamina $4 \mathrm{mg}+$ paracetamol $500 \mathrm{mg}$ + sulfato de pseudoefedrina $60 \mathrm{mg}$, clorfenamina $4 \mathrm{mg}$, hidróxido de aluminio $400 \mathrm{mg}+$ hidróxido de magnesio $400 \mathrm{mg}+$ simeticona activada $30 \mathrm{mg}$, 
Tabla 1. Proporción y caracteristicas de personas adultas que se automedican.

\begin{tabular}{|c|c|c|c|c|}
\hline Proporción y características & N & $\%$ & IC 95\% & Valor $p$ \\
\hline \multicolumn{5}{|l|}{ Se automedica $(n=433)$} \\
\hline Sí & 380 & 87,8 & 84,3 a 90,5 & \\
\hline No & 53 & 12,2 & 9,5 a 15,7 & \\
\hline Automedicación según sexo $(n=380)$ & & & & 0,103 \\
\hline Mujeres & 258 & 67,9 & 63,0 a 72,4 & \\
\hline Varones & 122 & 32,1 & 27,6 a 37,0 & \\
\hline Grupo de edad $(n=380)$ & & & & 0,651 \\
\hline 25 a 34 & 161 & 42,4 & 37,5 a 47,4 & \\
\hline 35 a 44 & 99 & 26,1 & 21,9 a 30,7 & \\
\hline 45 a 54 & 65 & 17,1 & 13,6 a 21,2 & \\
\hline Más de 55 & 55 & 14,5 & 11,3 a 18,4 & \\
\hline Grado de instrucción ( $n=380)$ & & & & $<0,000$ \\
\hline Superior & 163 & 42,9 & 38,0 a 47,9 & \\
\hline Secundaria & 148 & 38,9 & 34,2 a 43,9 & \\
\hline Primaria & 69 & 18,2 & 14,6 a 22,4 & \\
\hline Ocupación ( $n=380$ ) & & & & 0,05 \\
\hline Trabajador independiente & 190 & 50,0 & 45,0 a 55,0 & \\
\hline Ama de casa & 118 & 31,1 & 26,6 a 35,9 & \\
\hline Trabajador dependiente & 49 & 12,9 & 9,9 a 16,7 & \\
\hline Estudiante & 23 & 6,1 & 4,0 a 9,0 & \\
\hline Ingreso económico $(n=380)$ & & & & 0,05 \\
\hline Menor que el mínimo vital (< a \$ 245) & 232 & 61,1 & 56,1 a 65,8 & \\
\hline Mayor que el mínimo vital (> a \$245) & 148 & 39,0 & 34,2 a 43,9 & \\
\hline
\end{tabular}

Tabla 2. Principales razones de automedicación en personas adultas.

\begin{tabular}{|c|c|c|c|}
\hline Razones para la automedicación ( $n=380$ ) & N & $\%$ & IC $95 \%$ \\
\hline Mi enfermedad es leve & 350 & 92,1 & 88,9 a 94,4 \\
\hline Ya sé lo que recetan & 210 & 55,3 & 50,2 a 60,2 \\
\hline El costo de atención médica es elevado & 184 & 48,4 & 43,4 a 53,4 \\
\hline Los tratamientos ya son conocidos & 112 & 29,5 & 25,1 a 34,2 \\
\hline Utilizo las recetas anteriores & 89 & 23,4 & 19,4 a 27,9 \\
\hline $\begin{array}{l}\text { El tiempo de espera es prolongado en los servicios } \\
\text { de salud }\end{array}$ & 71 & 18,7 & 15,1 a 22,9 \\
\hline Me maltratan en los servicios de salud & 64 & 16,8 & 13,4 a 20,9 \\
\hline No tengo confianza en los médicos & 48 & 12,6 & 9,6 a16,4 \\
\hline \multicolumn{4}{|l|}{ Recomendaron la automedicación $(n=380)$} \\
\hline No, tomé por iniciativa propia & 86 & 22.6 & 18,7 a 27,1 \\
\hline Sí, me recomendaron $(n=294)$ & 294 & 77,4 & 70,1 a 78,9 \\
\hline El técnico en salud & 204 & 69,4 & 63,9 a 74,4 \\
\hline Mi familiar & 168 & 57,1 & 51,4 a 62,7 \\
\hline El químico farmacéutico & 89 & 30,3 & 25,3 a 35,8 \\
\hline \multicolumn{4}{|l|}{ Medios de influencia $(n=380)$} \\
\hline Televisión & 284 & 74,7 & 70,1 a 78,9 \\
\hline Radio & 186 & 48,9 & 44,0 a 54,0 \\
\hline Propaganda escrita & 84 & 22,1 & 18,2 a 26,6 \\
\hline Internet & 67 & 17,6 & 14,1 a 21,8 \\
\hline
\end{tabular}

omeprazol $20 \mathrm{mg}$, ranitidina $150 \mathrm{mg}$ y trimetoprima $160 \mathrm{mg}+$ sulfametoxazol $800 \mathrm{mg}$.

\section{DISCUSIÓN}

En la presente investigación se ha determinado una alta frecuencia de automedicación (87,8\%). En diversos estudios realizados en países de América Latina se hallaron cifras variables: en Chile, en pacientes que acudieron a un centro de atención ambulatoria $(85 \%)^{(8)}$; en México, en personas que acudieron a la farmacia comunitaria $(80,4 \%)^{(9)}$; en Ecuador, en estudiantes de enfermería (88\%) ${ }^{(10)}$; en Colombia, en adultos $(77,5 \%){ }^{(11)}$ y en universitarios $(97 \%){ }^{(12)}$. En países europeos se hallaron los siguientes resultados: en universitarias de España (90,8\%) ${ }^{(13)}$, en estudiantes mujeres de Malasia $(80,9 \%)^{(14)}$ y en el este y sur de Europa, la prevalencia de automedicación varió de 1 a 210 por $1,000{ }^{(15)}$. La OMS ha manifestado que la automedicación es válida en sociedades desarrolladas ${ }^{(1)}$. Sin embargo, que en países como Perú, la automedicación podría darse por la deficiente cobertura de los sistemas de seguridad social, por el sistema socioeconómico ${ }^{(16,17)}$ y el aumento de la publicidad de los medicamentos ${ }^{(18)}$.

Se ha encontrado predominancia de automedicación en el sexo femenino $(67,9 \%)$ y más frecuente en grupos con edades entre 25 a 34 años (42,4\%), resultado que es consistente con otros estudios. En España, el 72\% corresponde a la población femenina ${ }^{(19)}$; en Chile se encontró mayor proporción en mujeres y el $86 \%$ tenía entre 21 a 30 años ${ }^{(8)}$; en Colombia, 62,6\% eran mujeres y la edad media fue 44 años ${ }^{(11)}$. Sin embargo, estos resultados difieren con otros obtenidos en Ecuador, donde los estudiantes varones se automedicaron con mayor frecuencia $(80 \%){ }^{(20)}$. La explicación a este fenómeno es que generalmente las mujeres afrontan la enfermedad de manera diferente y poseen una mayor facilidad para reconocer el problema de salud; además prevalece su sentido intuitivo y de responsabili- 
Tabla 3. Medicamentos más usados para la automedicación por personas adultas con problemas de salud.

\begin{tabular}{|c|c|c|c|}
\hline Clasificación por actividad terapéutica & $\mathrm{N}$ & $\%$ & IC $95 \%$ \\
\hline Analgésicos y antipiréticos & 246 & 64,7 & 59,8 a 69,4 \\
\hline Antiinflamatorios y antirreumáticos no esteroideos & 214 & 56,3 & 51,3 a 61,2 \\
\hline Antihistamínicos para uso sistémico & 118 & 31,1 & 26,6 a 35,9 \\
\hline Antiácidos & 110 & 28,9 & 24,6 a 33,7 \\
\hline Agentes contra la úlcera péptica y el reflujo gastroesofágico & 84 & 22,1 & 18,2 a 26,6 \\
\hline Sulfamidas y trimetropima & 71 & 18,7 & 15,1 a 22,9 \\
\hline Quinolonas, antibacterianos & 67 & 17,6 & 14,1 a 21,8 \\
\hline Expectorantes, combinaciones con supresores de la tos & 61 & 16,1 & 12,7 a 20,1 \\
\hline Antibacterianos betalactámicos, penicilinas & 46 & 12,1 & 9,2 a 15,8 \\
\hline Drogas hipoglucemiantes orales & 38 & 10,0 & 7,3 a 13,5 \\
\hline Laxantes & 31 & 8,2 & 5,8 a 11,4 \\
\hline Vitaminas del complejo B & 28 & 7,4 & 5,1 a 10,5 \\
\hline \multicolumn{4}{|l|}{ Problemas de salud } \\
\hline Dolor & 246 & 64,7 & 59,8 a 69,4 \\
\hline Fiebre & 194 & 51,1 & 46,0 a 56,0 \\
\hline Gripe & 189 & 49,7 & 44,7 a 54,7 \\
\hline Alergia & 152 & 40,0 & 35,2 a 45,0 \\
\hline Ardor/dolor estomacal & 110 & 28,9 & 24,6 a 33,7 \\
\hline Infección & 104 & 27,4 & 23,1 a 32,1 \\
\hline Tos & 61 & 16,1 & 12,7 a 20,1 \\
\hline Diarrea & 38 & 10,0 & 7,3 a 13,5 \\
\hline Diabetes & 32 & 8,4 & 6,0 a 11,7 \\
\hline Estreñimiento & 31 & 8,2 & 5,8 a 11,4 \\
\hline Deficiencia de vitamina & 28 & 7,4 & 5,1 a 10,5 \\
\hline
\end{tabular}

dad ante su salud y de las personas de su entorno ${ }^{(21,22)}$. No obstante, las actitudes de cada género hacia el consumo de medicamentos no están relacionadas con una «masculinidad» $\mathrm{o} « f e m i-$ nidad», ya que los discursos médicos, antropológicos y la oferta médica han ido moldeando a lo largo de la historia los comportamientos de hombres y mujeres ${ }^{(23)}$.

Se halló que $42,9 \%$ tenía estudio superior y $50,0 \%$ tenía trabajo independiente con sueldo mínimo vital $(61,1 \%)$. De forma diferente, en Chile, se obtuvo que $44,5 \%$ tenía educación media completa y $28,5 \%$, educación superior ${ }^{(8)}$. Estas razones que evidencian un alto porcentaje de automedicación podrían significar que a mayor cultura faltó tiempo para consultar al médico $(18,2 \%)^{(8)}$. El resultado de este estudio podría haber sido porque la población tiene mayor sentimiento de autonomía personal en cuanto a decisiones sobre la propia salud ${ }^{(29)}$. Asimismo, la automedicación también depende en gran medida de las creencias, hábitos, mitos, desconocimiento y desinformación de la gente ${ }^{(16)}$. Pero, este comportamiento más que una aparente autosuficiencia responde a necesidades muy concretas como la falta de dinero, tiempo e ineficiencia de los servicios de salud ${ }^{(17)}$. Asimismo, un 17,8\% consideró que el tiempo de espera es prolongado en los servicios de salud; cercano porcentaje fue obtenido en Lima, Perú $(18,3 \%)^{(5)}$, lo cual genera un bajo nivel de satisfacción del usuario ${ }^{(30)}$. Esto podría deberse al desconocimiento de herramientas de gestión por parte del personal que dirige los centros de salud ${ }^{(31)}$.

Las personas se automedicaron en mayor medida por recomendaciones del personal técnico $(69,4 \%)$, dato superior a la recomendada por familiares en México (26,7\%). Esto podría deberse a que las personas asocian la enfermedad con los síntomas experimentados ${ }^{(9)}$.

La publicidad en televisión fue el medio más influyente $(74,7 \%)$; hallazgo similar fue encontrado en Colombia (70\%) ${ }^{(32)}$. Podríamos afirmar que los anuncios dirigidos al público en general deben contribuir a que la población pueda tomar decisiones racionales sobre la utilización de medicamentos ${ }^{(33)}$. Sin embargo, existe una serie de beneficios asociados a la revelación de información vía publicidad en materia de medicamentos que suelen ser obviados al proponer o defender una regulación como la que tiene el Perú ${ }^{(34)}$.

Se halló al igual que en la mayoría de los estudios, la predominancia del uso de analgésicos y antipiréticos (64,7\%), antiinflamatorios $(56,3 \%)$ y antihistamínicos (31,1\%). Ello se relacionó con las patologías más frecuentes como el dolor $(64,7 \%)$, fiebre $(51,1 \%)$ y gripe (49,7\%). En Honduras, el uso de AI- 
NEs representa el $81,81 \%{ }^{(35)}$; mientras que en países de Latinoamérica se ha encontrado porcentajes por debajo del $60 \%$; en Colombia, analgésicos y antipiréticos $(44,3 \%)^{(11)}$; en Brasil, $47,6 \%$ y $45,42 \%{ }^{(36,37)}$; en Ecuador, $46 \%$ y $31,1 \%$ consumen AINEs ${ }^{(10,20)}$, en el mismo país el 30\% usó antigripales y $18 \%$ tenía cefalea ${ }^{(11)}$; en Colombia, analgési$\cos (59,3 \%)$, tuvieron dolor $(31,1 \%)$ y gripe (20\%) ${ }^{(27)}$; en Chile, analgésicos $(26,45 \%)^{(8)}$. En países europeos y asiáticos también se hallaron porcentajes bajos; en España, utilizaron antiinflamatorios $48,7 \%$ y las enfermedades frecuentes fueron cefalea $(41,9 \%)$, dismenorrea $(38,1 \%)$, dolor $(21,9 \%)$ y procesos gripales $(18,1 \%)^{(19)}$; en Portugal, antinflamatorios $(1,4 \%){ }^{(38)}$; en Malasia, analgésicos y antipiréticos $(30,2 \%)^{(14)}$; en la India, antiinflamatorios $(27,2 \%){ }^{(28)}$; en Rusia, usaron principalmente analgésicos, antipiréticos, expectorantes y antigripales ${ }^{(39)}$. El hábito generalizado de consumo de analgésicos y antiinflamatorios podría generar otros problemas de salud debido a la presencia de reacciones adversas, tales como epigastralgia e hipertensión arterial ${ }^{(9,40)}$, siendo los más afectados los sistemas hematológico y gastrointestinal ${ }^{(41,42)}$.

El dolor fue el principal problema de salud, pero este representa los principales signos y síntomas de toda enfermedad, ya que el dolor es una experiencia sensorial y emocional, generalmente desagradable, que pueden experimentar todos aquellos seres vivos; por lo tanto, existe la necesidad de aplacarla con medicamentos $\mathrm{u}$ otros tratamientos que elija el paciente ${ }^{(43)}$.

La limitación fue que se realizó un estudio de automedicación en personas que acudieron a las boticas del distrito de Jesús Nazareno; por ende, estos resultados no reflejan la automedicación en la población en general, debido a que no muestra la situación de adultos que acudieron a otro tipo de establecimientos; además, no se ha considerado a personas mayores de 65 años.
Se recomienda realizar futuros estudios que reflejen la automedicación en diversos distritos con distintos grupos de edades de la población en general.

En conclusión, la automedicación continúa siendo un problema latente de salud pública en el DJN, el cual se manifestó en una alta frecuencia en la población adulta, predominando el sexo femenino. A mayor grado de instrucción, mayor automedicación. El principal problema de salud fue el dolor, mientras que el motivo de la automedicación obedeció a la levedad de la enfermedad. El tipo de medicamento de mayor uso fue el analgésico. En este sentido, es necesario que el Ministerio de Salud y Educación implementen estrategias educativas a nivel nacional con el fin de promover el uso responsable de medicamentos.

\section{AGRADECIMIENTOS}

A la Universidad Nacional San Cristóbal de Huamanga, alma mater de mi formación profesional, al Comité Institucional de Ética en Investigación (CIEI) del INS por la revisión y aprobación de la hoja de consentimiento informado y en especial a las personas encuestadas del DJN por el tiempo e información compartida.

\section{REFERENCIAS BIBLIOGRÁFICAS}

1. Organización Mundial de la Salud. El Papel del farmacéutico en el autocuidado y la automedicación. Reunión de Grupo Consultivo de la OMS y SAFYB. La Haya; 1998. Disponible en: http://goo. gl/iZR9VC.

2. Ley N 26842 (1997). Ley General de Salud. Diario Oficial El Peruano Año XV Nº 6232: 151245. Disponible en: https://goo.gl/exbl1s.

3. Ley N² 29459 (2009). Ley de Productos Farmacéuticos, Dispositivos Médicos y Productos Sanitarios. Diario Oficial El Peruano: 406565. Disponible en: http://goo.gl/713g8p.

4. Diario La Nueva. La automedicación puede resultar el peor remedio. 2014. Disponible en: http://goo. gl/iU8Cr5.

5. Martinez L. Percepción de la automedicación con antibióticos en los usuarios externos en un hospital público en Lima. [tesis de post grado]. Perú: Universidad Nacional Mayor de San Marcos; 2013:62 pp.

6. Guzmán L, Murrieta M. Factores que influyen en la automedicación en adultos de cuatro centros poblados de la cuenca media del río Nanay, dis- trito de San Juan Bautista. [tesis de post grado]. Universidad Nacional de la Amazonia Peruana; 2010:77 pp.

7. Acción Internacional para la Salud Perú. Consumers International: Transnacionales farmacéuticas transgreden las normas éticas. 2007. Disponible en: http://goo.gl/QBxW3z.

8. Moya S. Estudio prevalencia de automedicación en consultantes a un centro de atención ambulatoria adosado a un hospital de una comuna de Santiago. [Tesis de pre grado]. Universidad de Chile; 2012:58 pp.

9. Galar M, Amaya Ch, Gómez O, Carmona Z, Téllez L. Estudio de automedicación en una farmacia comunitaria de la ciudad de Toluca. Revista Mexicana de Ciencias Farmacéuticas. 2009; 40:1:405-11 Disponible en: http://goo.gl/wyQGXI.

10. Morillo M, Valencia V. Factores relacionados con la automedicación en los estudiantes de enfermería de la facultad ciencias de la salud de la universidad Técnica del Norte, Ecuador; 2010. Disponible en: http://goo.gl/f477×3.

11. Machado J, Echeverri L, Londoño M, Moreno P, Ochoa S, Ruiz J. Factores económicos, sociales y culturales asociados con la automedicación. Universidad Tecnológica de Pereira, Colombia. Biomédica Rev Inst Nac Salud. 2014;34:580-8. DOI: http://dx.doi.org/10.7705/biomedica.v34i4.2229.

12. Tobón F. Estudio sobre automedicación en la Universidad de Antioquia, Colombia. IATREIA 2012; 15:4:242-47. Disponible en: http://goo.gl/JrOheo.

13. Guillen P, Francès F, Gimenez F, Sáiz C. Estudio sobre automedicación en población universitaria española. Universitat de València. Rev Clin Med Fam 2010; 3:2:99-103. Disponible en: http://goo. gl/C4goKv.

14. Sohair E, Mohamed I, Subish P. Medication storage and self-medication behaviour amongst female students in Malaysia. Pharmacy Practice, 2010;8(4):226-32. Disponible en: http://goo.gl/ uiM6Wv.

15. Grigoryan L, Haaijer F, Burgerhof J, Mechtler R, Deschepper R, Tambic A, et al. Self-medication with antimicrobial drugs in Europe. Emerging Infectious Dis. 2006;12(3):452-59. DOI: 10.3201/ eid1203.050992.

16. Cuvi J. Automedicación y crisis del modelo biomédico. Rev Fac Ciencias Médicas, Universidad de Cuenca, Ecuador. 2012;30(2):40-3. Disponible en: http://goo.gl/LvpxKK.

17. Cuvi J. La enfermedad silenciosa. Una mirada social a la automedicación en el Ecuador. Corporación Acción Vital: Ecuador. Rev Fac Cien Med Quito, 2011;36(1):40.

18. Laporte J, Castel J. El médico ante la automedicación. Medical Clínica Barcelona. 1992;99:414-6.

19. Álvarez J. Prevalencia de automedicación en estudiantes de farmacia y medicina de la Universidad de Granada. [tesis de máster]. España; 2008:43 pp.

20. Almeida M. Prevalencia y prácticas de automedicación en los estudiantes de Primero a Quinto año de la Facultad de Medicina de la Universidad San Francisco de Quito. [Tesis de grado]. Ecuador; 2009:43 pp. Disponible en: http://goo.gl/7cfKg3.

21. Mirón C, Molina M, Tardido C. Automedicación, ¿Cuestión de género? Un estudio exploratorio en la ciudad autónoma de Melilla. Universidad de Granada, España, 2005. Disponible en: https:// goo.gl/CQ1kcE.

22. Vega A, Romo N, Markez I, Poo M, Meneses C, Gil E. Mujeres y psicofármacos: las voces y los ecos. Un estudio comparativo en tres Comunida- 
des Autónomas de España. Instituto de la Mujer. Disponible en: http://goo.gl/Glk06l.

23. Dinges M. La investigación histórica y el debate actual sobre la salud masculina: el caso de Alemania. 2014;66(1):p040. DOI: http://dx.doi.org/10.3989/ asclepio.2014.14.

24. Figueiras A, Caamano F, Gestal J. Sociodemographic factors related to self-medication in Spain. Eur J Epidemiol. 2000:16:19-26. Disponible en: http:// goo.gl/4dnXYu.

25. Caamaño F, Figueiras A, Lado E, Gestal J. La au tomedicación: concepto y perfil de sus "usuarios". Gac Sanit. 2000;14(4):294-9. Disponible en: http:// goo.gl/D9oaE1.

26. Llanos L, Contreras C, Velásquez J, Mayca J, Lecca L, Reyes R y col. Automedicación en cinco provincias de Cajamarca Perú. Rev Med Hered. 2001;12(4):127-33. Disponible en: http://goo.gl/ Xn4dB7.

27. López J, Dennis R, Moscoso S. Estudio sobre la automedicación en una localidad de Bogotá, Colombia. Rev Salud Pública. 2009;11(3):432-42. Disponible en: http://goo.gl/0HfG45.

28. Selvaraj K, Kumar S, Ramalingam A. Prevalence of self-medication practices and its associated factors in Urban Puducherry, India. Institute of Post Graduate Medical Education and Research. Perspectives in Clinical Research. 2014;5:32-6. DOI: 10.4103/2229-3485.124569.

29. Rocha A, Harter R, Rotenberg L. Automedicación entre los trabajadores de enfermería de hospitales públicos, Brasil. Rev Latino-Am Enfermagem. 2009;17:6. http://dx.doi.org/10.1590/S010411692009000600014

30. Gutierrez E, Ramos W, Uribe M, Ortega-Loayza A Torres C, Montesinos D y col. Tiempo de espera y su relación con la satisfacción de los usuarios en la farmacia central de un Hospital General de Lima. Rev Perú Med Exp Salud Pública. 2009;26(1):61-5.

31. Alva G. La gestión de instituciones de salud en el Perú. Conexión ESAN; 2015. Disponible en: http:// goo.gl//zteKL.
32. Vacca C, Vargas C, Cañás M, Reveiz M. Publicidad y promoción de medicamentos: regulaciones y grado de acatamiento en cinco paises de América Latina, Colombia. Rev Panam Salud Pública. 2011;29(2):76-83. Disponible en: http://goo.gl/ $\mathrm{K} 221 \mathrm{iH}$.

33. Criterios éticos para la promoción de medicamentos. 1985. Disponible en: http://goo.gl/PWWUj2.

34. Rodríguez G. Mal diagnóstico: Las contraindicaciones de la prohibición de publicidad de productos farmacéuticos. Rev Economia y Derecho, UPC Perú. 2012;9:36.

35. Paz J, Aguilar E, Izaguirre S, Duran A. Automedicación en estudiantes de la carrera de medicina, Facultad de Ciencias Médicas, UNAH Honduras. Rev Fac Cienc Méd. 2013;31-6. Disponible en: http://goo.gl/24ciC9.

36. Martinez J, Farina G, Martinelli L, Nunes R, llias D, Moretti L. Original article study of self-medication for musculoskeletal pain among nursing and medicine students at Pontificia Universidad de Católica - São Paulo. Rev Bras Reumatol. 2014;54(2):90-4. Disponible en: http://goo.gl/mchM4i.

37. Rodríguez F, Aparecido R, Smokou A, Lascane $E$, Andrioli R, Fernandes $E$, et al. Frequência de automedicação entre acadêmicos de faculdade de medicina. Universidade Metropolitana de Santos. Brasil. 2010;15(2):53-7. Disponible en: http:// goo.gl/ZOXiJZ.

38. Gama H, Oliveira L, Pereira M, Azevedo A, Lunet N. Use of medicines by homeless people in Porto, Portugal. Cad Saúde Pública, Rio de Janeiro. 2014;30(1):207-12, Disponible en: http://goo. $\mathrm{gl} / \mathrm{OvmRqQ}$

39. Trofimova E. La influencia de la publicidad en el desarrollo del mercado de productos farmacéuticos OTC. Revista "Marketing en Rusia y en el extranjero" 2005;4. Disponible en: http://goo.gl/AOYJ7L.

40. Jiménez G, Debesa F, Bastanzuri T, Pérez J, Ávila J. Comportamiento de las reacciones adversas a los analgésicos y antiinflamatorios no esteroideos notificadas por el Sistema Cubano de Farmacovi- gilancia. Rev Cubana Farm. 2003;37:3. Disponible en: http://goo.gl/XU6t1R.

41. Bolesta S, Haber SL. Hepatotoxicity asociated with chronic acetaminophen administration in patients wthout risc factors. Ann Pharmacother 2002;36:331-3. DOI: 10.1345/aph.1A035.

42. Sánchez L, Hernández F. Reacciones adversas por antiinflamatorios no esteroideos. Rev Cubana de Farm. 2011;45(1):60-8. Disponible en: http:// goo.gl/1pu3Ke.

43. López F. Definición y clasificación del dolor. Clin Urol Complutense, Madrid. 1996:4:49-55.

Artículo recibido el 19 de mayo de 2016 y aceptado para publicación el 9 de agosto de 2016.

Fuente de financiamiento: Personal.

Conflicto de intereses: El autor declara no tene conflicto de interés.

Declaración: El material contenido en el manuscrito no ha sido publicado previamente o remitido a otra revista biomédica.

Se obtuvo consentimiento de las personas estudiadas en la investigación.

Correspondencia:

Mg. Mery Luz Pillaca Medina.

Dirección: Condominio Los Álamos Dpto. H-204, El Agustino, Lima 4, Perú.

Teléfono: 966-667980.

Correo electrónico: qf.meryluz@hotmail.com 